\title{
Wavelength Tunable Source of Subpicosecond Pulses from CW Passively Mode-Locked Two-Section Multiple-Quantum-Well Laser
}

\author{
Randal A. Salvatore, Thomas Schrans, and Amnon Yariv
}

\begin{abstract}
A wavelength tunable passively mode-locked semiconductor laser source of subpicosecond pulses is demonstrated. The system includes a two-section multiple-quantum-well laser which is coupled to an external grating for tuning and is followed by an external grating pair for pulse compression. $\mathbf{A}$ tuning range of $16 \mathrm{~nm}$ around $846 \mathrm{~nm}$ is obtained, resulting in compressed pulse widths as short as $260 \mathrm{fs}$ and pulse widths shorter than 600 fs for all wavelength values within this tuning range. Time bandwidth products are one to two times the transform limit.
\end{abstract}

$\mathrm{T}$

HE usefulness of a mode-locked laser is limited in many applications by its inability to produce an output centered at a desired wavelength. It has previously been demonstrated that a semiconductor laser coupled to an external cavity and followed by an external grating compressor can be used to generate stable pulse trains of subpicosecond pulse widths [1]-[6]. Wavelength tunability of passively mode-locked semiconductor lasers has also been demonstrated with tuning ranges up to $26 \mathrm{~nm}$ around $0.84 \mu \mathrm{m}$ [7]. So far, wavelength tunable mode-locked semiconductor lasers have been limited to pulse widths longer than 1 ps [7]-[10]. In this letter we present results combining broad-band wavelength tunability and subpicosecond pulse generation to create a tunable subpicosecond passively mode-locked semiconductor laser source.

The laser device and setup are similar to that described in [3], [7] where a grating $(600$ lines $/ \mathrm{mm})$ is used to form one end of the external cavity while the laser's high reflection coated $(90 \%)$ absorber facet serves as the other end of the external cavity (Fig. 1). The laser is a two-section four-quantum well laser with the gain facet antireflection (AR) coated to less than $5 \%$. The gain section is biased with a dc current source, and the absorber section is grounded for passively mode-locked operation. The light exiting the absorber is then compressed to subpicosecond pulse widths using a dual grating compressor as shown in Fig. 1. After compression, the light is either directed into a monochromator to measure the optical spectrum or a second harmonic collinear intensity auto-

Manuscript received March 5, 1993; revised April 14, 1993. This work was supported by the National Science Foundation, the Office of Naval Research, the Air Force Office of Scientific Research, and DARPA

The authors are with the Department of Applied Physics 128-95, California Institute of Technology, Pasadena, CA 91125.

IEEE Log Number 9210042.

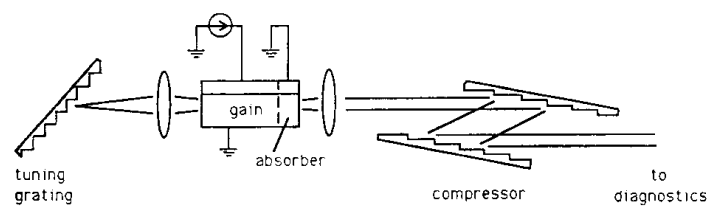

Fig. 1. Wavelength tunable two-section laser passively mode-locked in external cavity with external grating pair compressor.

correlator to determine the pulse width. A high-speed detector and microwave spectrum analyzer are used to monitor that the laser remains in the first harmonic of the external cavity which gives a repetition rate of $603 \mathrm{MHz}$ [11]. Throughout the experiment, the laser mount is maintained at $21^{\circ} \mathrm{C} \pm 0.5^{\circ} \mathrm{C}$.

By rotating the grating to select the wavelength of maximum feedback into the laser, the center wavelength of the optical spectrum can be tuned from 838 to $854 \mathrm{~nm}$, a range of $16 \mathrm{~nm}$. The mode-locking threshold for first harmonic operation over this range is shown in Fig. 2. The operating points above this threshold, where measurements were performed, are shown as squares in Fig. 2. For each of these wavelengths, the grating compressor is adjusted to minimize the compressed pulse width.

Using the second harmonic collinear intensity autocorrelator, pulse widths were measured at the indicated operating points (Fig. 2). The measured intensity autocorrelations are shown in Fig. 3, and the corresponding deconvolved pulse widths (assuming a sech ${ }^{2}$ profile) are shown in Fig. 4. The shortest pulses are obtained for wavelengths near the center and toward the longer wavelengths of the tuning range. Tuning toward the shorter wavelengths resulted in broader, yet still subpicosecond pulse widths with long tails. These long tails could not be eliminated by changing the compressor grating spacing, and are attributed to the presence of a nonlinear chirp component. Generally, the chirp on the pulses exiting the cavity is extremely linear, allowing compression by about a factor of ten, whereas the long tails are only visible after most of the linear chirp is removed. The pulses exiting the laser possess an upchirp, meaning the frequency increases within each pulse, and the magnitude of chirp is typically 


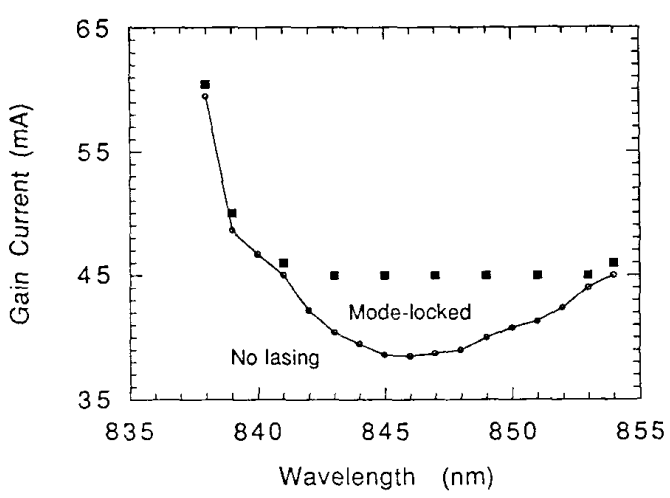

Fig. 2. Mode-locking range (solid line) as a function of gain current and wavelength. Autocorrelations and optical spectrum measurements were performed at currents and wavelengths indicated by squares.

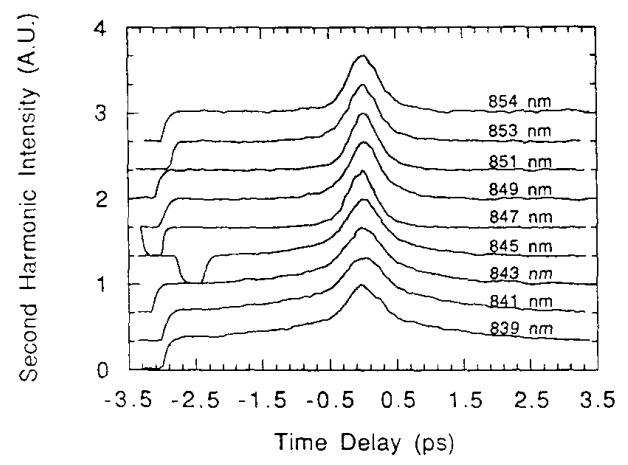

Fig. 3. Intensity autocorrelations at indicated wavelengths.

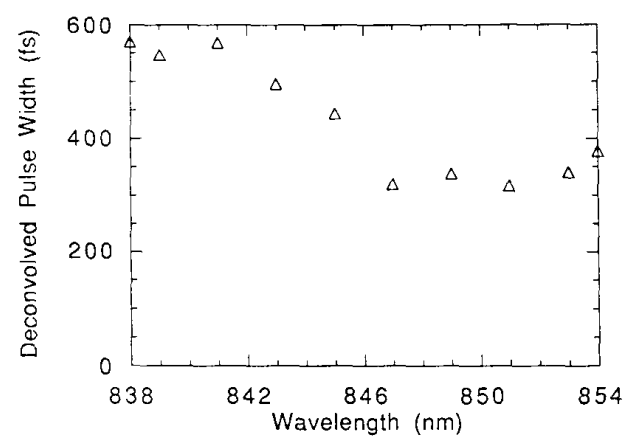

Fig. 4. Deconvolved pulse FWHM versus wavelength.

on the order of $0.6 \mathrm{ps} / \mathrm{nm}$. Measured pulse widths before compression are between 2 and 5 ps with the shorter uncompressed pulses occurring when tuning to shorter wavelengths. However, these shorter wavelength pulses measured possess the highest peak powers within the cavity, have the least linear chirp, and result in the longest pulses after compression. Increasing the current at shorter wavelengths does not reduce the pulse width considerably.

The optical spectrum under mode-locked operation is found to vary significantly upon tuning. For shorter wave-

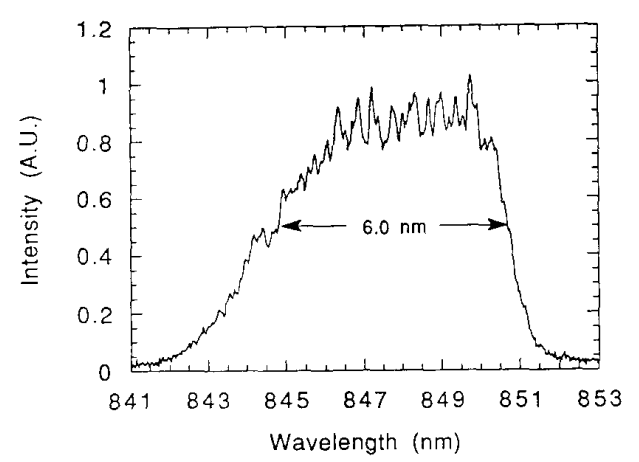

Fig. 5. Optical spectrum of mode-locked laser corresponding to minimum pulse width at $53 \mathrm{~mA}$ and centered at $848 \mathrm{~nm}$.

TABLE I

Comparison of Measlrements at Three Different Wavelengths FROM THE TUNABLE SUbPICOSECOND SEMICONDUCTOR LASER

\begin{tabular}{ccccccccc}
\hline$\lambda(\mathrm{nm})$ & $I_{\text {th }}(\mathrm{mA})$ & $I_{\text {meas }}(\mathrm{mA})$ & $\tau_{p}(\mathrm{fs})$ & $\begin{array}{c}\Delta \lambda \\
(\mathrm{nm})\end{array}$ & $\tau_{p} \Delta \nu$ & $\begin{array}{c}\text { Average } \\
\text { Power } \\
(\mu \mathrm{W})\end{array}$ & $\begin{array}{c}\text { Peak } \\
\text { Power } \\
(\mathrm{mW})\end{array}$ \\
\hline 839 & 48.8 & 50.0 & 550 & 3.0 & 0.69 & 36 & 38 \\
847 & 38.5 & 45.0 & 320 & 3.3 & 0.44 & 37 & 67 \\
853 & 43.4 & 45.0 & 370 & 3.6 & 0.50 & 24 & 38
\end{tabular}

lengths, the spectrum is nearly symmetric, while for longer wavelengths it develops a much sharper drop on the long wavelength side (Fig. 5). A comparison of the measurements at three different operating points within the tuning range is shown in Table I. Typical pulses are around $400 \mathrm{fs}$ FWHM and have time bandwidth products between one and two times the transform limit. Measured peak powers were small (typically $50 \mathrm{~mW}$ ) due to the $90 \%$ reflection at the output facet, and the $65 \%$ loss in the compressor.

A minimum pulse width, $\tau_{p}$, of $260 \mathrm{fs}$ is measured at a wavelength of $848 \mathrm{~nm}$ and a gain current of $53 \mathrm{~mA}$ with the laser mode-locked in the first harmonic. The higher current and cavity tuning result in an optical spectrum FWHM of $6.0 \mathrm{~nm}$, significantly broader than the spectra at lower currents (Table I). The optical spectrum for this shortest pulse is shown in Fig. 5, and the corresponding autocorrelation is shown in Fig. 6. The pulse peak power is found to be just over $100 \mathrm{~mW}$. The calculated timebandwidth product gives 0.65 (about 1.5 to 2 times transform limit). Due to the relatively high AR coating (5\%) of the gain facet, satellite pulses are observed with an energy of less than $10 \%$ of the main pulse [3], [7]. Wavelength tuning of mode-locked second and third harmonic subpicosecond pulses is also observed; however, higher harmonic operation results in longer compressed pulses when achieved at similar current levels.

In conclusion, broad-band wavelength tunability (16 nm) with subpicosecond pulses was demonstrated for an external cavity mode-locked semiconductor laser. Pulse widths below 600 fs after external compression with a grating pair were generated with a minimum pulse width 


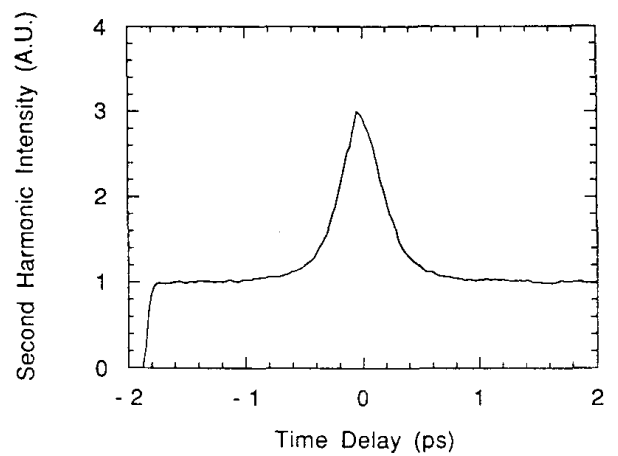

Fig. 6. Minimum pulse width autocorrelation for gain current of $53 \mathrm{~mA}$ tuned to $848 \mathrm{~nm}$.

of 260 fs $\left(\tau_{p} \Delta \nu=0.65\right)$ when the laser was operating at higher currents. Pulses were one to two times transform limited with long tails in the autocorrelations at the short wavelength side of the tuning range.

\section{ACKNOWLEDGMENT}

We would like to acknowledge both Ortel Corporation, Alhambra CA, for providing the laser device used in this experiment and S. Sanders for helpful discussions.

\section{REFERENCES}

[1] J. P. van der Ziel, W. T. Tsang, R. A. Logan, R. M. Mikulyak, and W. M. Augustyniak, "Subpicosecond pulses from passively modelocked GaAs buried optical guide semiconductor lasers," Appl. Phys. Lett., vol. 39, pp. 525-527, Oct. 1981.
[2] Y. Silberberg, and P. W. Smith, "Subpicosecond pulses from a mode-locked semiconductor laser," IEEE J. Quantum Electron., vol. 22, pp. 759-761, June 1986.

[3] T. Schrans, R. A. Salvatore, S. Sanders, and A. Yariv, "Subpicosecond (320 fs) pulses from CW passively mode-locked external cavity two-section multiquantum well lasers," Electron. Lett., vol. 28, pp. 1480-1482, July 1992.

[4] P. J. Delfyett, L. Florez, N. Stoffel, T. Gmitter, N. Andreadakis, G. Alphonse, and W. Ceislik, "200-fs, optical pulse generation and intracavity evolution in a hybrid mode-locked semiconductor diode-laser/amplifier system," Opt. Lett., vol. 17, pp. 670-672, May 1992.

[5] R. J. Helkey, D. J. Derickson, A. Mar, J. G. Wasserbauer, J. E. Bowers, and R. L. Thornton, "Colliding pulse effects in modelocked semiconductor diode lasers," presented at Conf. Lasers Electro-Optics, 1992, vol. 12, OSA Tech. Dig. Series, p. 408, Opt. Soc. Amer., Washington, DC, May 1992.

[6] N. Stelmakh and J. M. Lourtioz, " $230 \mathrm{fs,}, 25 \mathrm{~W}$ pulses from conventional mode-locked laser diodes with saturable absorber created by ion implantation," Electron. Lett., vol. 29, pp. 160-162, Jan. 1993.

[7] T. Schrans, S. Sanders, and A. Yariv, "Broad-band wavelength tunable picosecond pulses from $\mathrm{CW}$ passively mode-locked twosection multiple quantum-well lasers," IEEE Photon. Technol. Lett., vol. 4, pp. 323-326, Apr. 1992.

[8] M. C. Wu, Y. K. Chen, T. Tanbun-Ek, R. A. Logan, and M. A. Chin, "Tunable monolithic colliding pulse mode-locked quantum well lasers," IEEE Photon. Technol. Lett., vol. 3, pp. 874-876, Oct. 1991.

[9] J. M. Wiesenfeld, M. Kuznetsov, and A. S. Hou, "Tunable picosecond pulse generation using a compressed mode locked laser diode source," IEEE Photon. Technol. Lett., vol. 2, pp. 319-321, May 1990.

[10] M. Serenyi, J. Kuhl, and E. O. Göbel, "Pulse shortening of actively mode-locked diode lasers by wavelength tuning," Appl. Phys. Lett., vol. 50, pp. 1213-1215, May 1987.

[11] S. Sanders, A. Yariv, J. Paslaski, J. E. Ungar, and H. A. Zarem, "Passive mode locking of a two-section multiple quantum well laser at harmonics of the cavity round-trip frequency," Appl. Phys. Lett., vol. 58, pp. 681-683, Feb. 1991.

\title{
Output Coupling Induced Wavelength Shifts in Erbium-Doped Fiber Lasers
}

\author{
T. Rosadiuk, Member, IEEE, and J. Conradi, Senior Member, IEEE
}

\begin{abstract}
We present experimental results of shifting the operating wavelength of erbium fiber lasers by varying the wavelength independent output coupling. No wavelength selective elements are incorporated in the laser cavity. The results are in agreement with a simplified theory as previously published by others.
\end{abstract}

\section{INTRODUCTION}

$S^{\mathrm{E}}$ EVERAL methods for obtaining tunable operation of erbium-doped fiber lasers have been reported, all of which require some form of wavelength selective element

Manuscript received February 2, 1993; revised Aprit 15, 1993. This work was supported by the Natural Sciences and Engineering Research Council of Canada, Bell Northern Research Limited and TRLabs through the NSERC/BNR/TRLabs Chair in Fiber Optic Communications at the University of Alberta.

The authors are with TRLabs, Edmonton. Alta., Canada T5K 2P7.

IEEE Log Number 9209847. as part of the laser cavity. Typically, intracavity filters or gratings, as well as output couplers that have wavelength dependent reflectivity have been used to obtain broadband tuning and very narrow line operation of ring lasers as well as other laser configurations [1]-[8].

Recently, a simplified theory of fiber laser operation has been published [9] in which it is predicted that the wavelength of erbium-doped fiber lasers can be tuned within and between wavelength bands simply by varying the wavelength independent reflectivity of the output coupling mirror. In this letter we report on experiments in which an erbium-doped fiber laser is wavelength tuned within and between three narrow wavelength bands using only the variable output coupling of one output mirror, taking extreme care to ensure that none of the components within the laser cavity has a significant wavelength dependence that could cause the laser to be wavelength 